University of Texas at El Paso

ScholarWorks@UTEP

$1-2018$

\title{
How to Detect Crisp Sets Based on Subsethood Ordering of Normalized Fuzzy Sets? How to Detect Type-1 Sets Based on Subsethood Ordering of Normalized Interval-Valued Fuzzy Sets?
}

\author{
Christian Servin \\ El Paso Community College, cservin@gmail.com \\ Olga Kosheleva \\ The University of Texas at El Paso, olgak@utep.edu \\ Vladik Kreinovich \\ The University of Texas at El Paso, vladik@utep.edu
}

Follow this and additional works at: https://scholarworks.utep.edu/cs_techrep

Part of the Computer Sciences Commons

Comments:

Technical Report: UTEP-CS-18-01

\section{Recommended Citation}

Servin, Christian; Kosheleva, Olga; and Kreinovich, Vladik, "How to Detect Crisp Sets Based on Subsethood Ordering of Normalized Fuzzy Sets? How to Detect Type-1 Sets Based on Subsethood Ordering of Normalized Interval-Valued Fuzzy Sets?" (2018). Departmental Technical Reports (CS). 1217. https://scholarworks.utep.edu/cs_techrep/1217

This Article is brought to you for free and open access by the Computer Science at ScholarWorks@UTEP. It has been accepted for inclusion in Departmental Technical Reports (CS) by an authorized administrator of ScholarWorks@UTEP. For more information, please contact Iweber@utep.edu. 


\section{How to Detect Crisp Sets Based on Subsethood Ordering of Normalized Fuzzy Sets? How to Detect Type-1 Sets Based on Subsethood Ordering of Normalized Interval-Valued Fuzzy Sets?}

\author{
Christian Servin \\ Computer Science and Information \\ Technology Systems Department \\ El Paso Community College \\ 919 Hunter, El Paso, Texas 79915, USA \\ cservin@gmail.com
}

\author{
Olga Kosheleva and Vladik Kreinovich \\ University of Texas at El Paso \\ $500 \mathrm{~W}$. University \\ El Paso, TX 79968, USA \\ olgak@utep.edu,vladik@utep.edu
}

\begin{abstract}
If all we know about normalized fuzzy sets is which set is a subset of which, will we be able to detect crisp sets? It is known that we can do it if we allow all possible fuzzy sets, including non-normalized ones. In this paper, we show that a similar detection is possible if we only allow normalized fuzzy sets. We also show that we can detect type- 1 fuzzy sets based on the subsethood ordering of normalized interval-valued fuzzy sets.
\end{abstract}

\section{INTRODUCTION}

A fuzzy set is usually defined as function $A$ from a certain set $U$ - known as Universe of discourse - to the interval [0,1]; see, e.g., [1], [2], [3], [5], [6], [8]. Traditional - "crisp" - sets can be viewed as particular cases of fuzzy sets, for which $A(a) \in\{0,1\}$ for all $x$.

In most applications, we consider normalized fuzzy sets, i.e., fuzzy sets for which $A(x)=1$ for some $x \in U$. For crisp sets, this corresponds to considering non-empty sets.

For two crisp sets, $A$ is a subset or $B$ if and only if $A(x) \leq$ $B(x)$ for all $x$. The same condition is used as a definition of the subsethood ordering between fuzzy sets: a fuzzy set $A$ is a subset of a fuzzy set $B$ if $A(x) \leq B(x)$ for all $x$. Subsets $B \subseteq A$ which are different from the set $A$ are called proper subsets of $A$.

A natural question is: if we have a class of all normalized fuzzy sets with the subsethood relation, can we detect which of these fuzzy sets are crisp? It is known that:

- if we alow all possible fuzzy sets - even non-normalized ones,

- then we can detect crisp sets; see, e.g., [7].

In this paper, we show that such a detection is possible even if we restrict ourselves only to normalized sets.

\section{Main Result}

In order to describe general crisp sets in terms of subsethood relation $\subseteq$ between fuzzy sets, we will first describe some auxiliary notions in these terms.
In this section, we only consider normalized fuzzy sets.

Proposition 1. A normalized fuzzy set is a 1-element crisp set if and only if it has no proper normalized fuzzy subsets, i.e., if and only if $B \subseteq A$ implies $B=A$.

Proof.

$1^{\circ}$. Let us first prove that a 1 -element crisp set $A=\left\{x_{0}\right\}$ (i.e., a set for which $A\left(x_{0}\right)=1$ and $A(x)=0$ for all $x \neq x_{0}$ ) has the desired property.

Indeed, if $B \subseteq A$, this means that $B(x) \leq A(x)$ for all $x$. For $x \neq x_{0}$, we have $A(x)=0$, so we have $B(x)=0$ as well.

Since $B$ is a normalized fuzzy set, it has to attain value 1 somewhere. Since we have $B(x)=0$ for all $x \neq x_{0}$, the only point $x \in U$ at which we can have $B(x)=1$ is the point $x_{0}$. Thus, we have $B\left(x_{0}\right)=1$.

So, indeed, we have $B(x)=A(x)$ for all $x$, i.e., $B=A$.

$2^{\circ}$. Vice versa, let us prove that each normalized fuzzy set $A$ which is different from a 1-element crisp set has a proper normalized fuzzy subset.

Indeed, since $A$, is normalized, we have $A\left(x_{0}\right)=1$ for some $x_{0}$. Then, we can take $B=\left\{x_{0}\right\}$. Clearly, $B \subseteq A$, and, since $A$ is not a 1-element crisp set, $B \neq A$.

The proposition is proven.

Definition 1. By a 2-element set, we mean a normalized fuzzy set $A$ for which $A(x)>0$ for exactly two elements $x \in U$.

Proposition 2. For a normalized fuzzy set $A$ which is not a 1 element crisp set, the following two conditions are equivalent to each other:

- $A$ is a non-crisp 2-element set, and

- the class $\{B: B \subseteq A\}$ of all its subsets is linearly ordered, i.e.:

$$
\text { if } B_{1} \subseteq A \text { and } B_{2} \subseteq A \text { then } B_{1} \subseteq B_{2} \text { or } B_{2} \subseteq B_{1} \text {. }
$$




\section{Proof.}

$1^{\circ}$. Let us first prove that if $A$ is a 2-element non-crisp set, then the class of all its subsets is linearly ordered.

Indeed, since $A$ is a normalized fuzzy set, we must have $A\left(x_{0}\right)=1$ for some $x_{0} \in U$. Since $A$ is a 2-element set, there must be one more value $x \in U$ for which $A(x)>0$. Let us denote this value by $x_{1}$. So, we have:

- $A\left(x_{0}\right)=1$,

- $A\left(x_{1}\right)>0$ and

- $A(x)=0$ for all other $x \in U$.

If we had $A\left(x_{1}\right)=1$, then $A$ would be a crisp set - namely, we would have $A=\left\{x_{0}, x_{1}\right\}$. Since $A$ is a non-crisp set, we thus cannot have $A\left(x_{1}\right)=1$, so we have $0<A\left(x_{1}\right)<1$.

If $B$ is a normalized fuzzy set for which $B \subseteq A$, then for all $x$ different from $x_{0}$ and $x_{1}$, we have $B(x) \leq A(x)=0$ and thus, $B(x)=0$. Since $B$ is normalized, we have $B(x)=1$ for some $x$.

- This $x$ cannot be different from $x_{0}$ and $x_{1}$ - because then $B(x)=0$.

- This $x$ cannot be equal to $x_{1}$, since then we would have $1=B\left(x_{1}\right) \leq A\left(x_{1}\right)<1$ and $1<1$.

Thus, this $x$ must be equal to $x_{0}$, i.e., we must have $B\left(x_{0}\right)=$ 1. So, all fuzzy normalized subsets $B$ of the set $A$ have the following form:

- $B\left(x_{0}\right)=1$,

- $B\left(x_{1}\right) \leq A\left(x_{1}\right)$, and

- $B(x)=0$ for all other $x$.

For two such subsets, we can have:

- either $B_{1}\left(x_{1}\right) \leq B_{2}\left(x_{1}\right)$,

- or $B_{2}\left(x_{1}\right) \leq B_{1}\left(x_{1}\right)$.

One can easily check that:

- if $B_{1}\left(x_{1}\right) \leq B_{2}\left(x_{1}\right)$, then $B_{1}(x) \leq B_{2}(x)$ for all $x$ and thus, $B_{1} \subseteq B_{2}$;

- similarly, if $B_{2}\left(x_{1}\right) \leq B_{1}\left(x_{1}\right)$, then $B_{2}(x) \leq B_{1}(x)$ for all $x$ and thus, $B_{2} \subseteq B_{1}$.

So, for every two normalized fuzzy subsets $B_{1}$ and $B_{2}$ of the set $A$, we have either $B_{1} \subseteq B_{2}$ or $B_{2} \subseteq B_{1}$. Thus, the class of all such subsets is indeed linearly ordered.

$2^{\circ}$. To complete the proof of Proposition 2, let us now prove that if a normalized fuzzy set $A$ is not a 1-element fuzzy set and not a non-crisp 2-element set, then the class

$$
\{B: B \subseteq A\}
$$

is not linearly ordered, i.e., there exists normalized fuzzy subsets $B_{1} \subseteq A$ and $B_{2} \subseteq A$ for which $B_{1} \nsubseteq B_{2}$ and $B_{2} \not \subset B_{1}$.

The fact that the set $A$ is not a 1-element set means that $A(x)>0$ for at least two different values $x$.

By definition, a non-crisp 2-element set is a normalized fuzzy set:

- which is a 2-element set and

- which is not crisp.
So, if a normalized fuzzy set $A$ is not a non-crisp 2-element set, this means that it is:

- either not a 2-element set

- or it is a crisp 2-element set.

Let us show that in both cases, we can find subsets $B_{1} \subseteq A$ and $B_{2} \subseteq A$ for which $B_{1} \not \subset B_{2}$ and $B_{2} \not \subset B_{1}$.

$2.1^{\circ}$. Let us first consider the case when $A$ is not a 2-element set, i.e., when, in addition to the point $x_{0}$ at which $A\left(x_{0}\right)=$ 1 , there exist at least two other points $x_{1}$ and $x_{2}$ for which $A\left(x_{1}\right)>0$ and $A\left(x_{1}\right)>0$.

In this case, we can take the following sets $B_{1}$ and $B_{2}$ :

- $B_{1}\left(x_{0}\right)=B_{2}\left(x_{0}\right)=1$;

- $B_{1}\left(x_{1}\right)=A\left(x_{1}\right)$ and $B_{2}\left(x_{1}\right)=0$;

- $B_{2}\left(x_{1}\right)=0$ and $B_{2}\left(x_{2}\right)=A\left(x_{2}\right)$, and

- $B_{1}(x)=B_{2}(x)$ for all other $x$.

One can see that $B_{1}(x) \leq A(x)$ and $B_{2}(x) \leq A(x)$ for all $x$, so indeed $B_{1} \subseteq A$ and $B_{2} \subseteq A$. However, here:

- $B_{1}\left(x_{1}\right)=A\left(x_{1}\right)>0=B_{2}\left(x_{1}\right)$, so we cannot have $B_{1} \subseteq B_{2}$, because that would imply $B_{1}\left(x_{1}\right) \leq B_{2}\left(x_{1}\right)$;

- similarly, $B_{2}\left(x_{2}\right)=A\left(x_{2}\right)>0=B_{1}\left(x_{2}\right)$, so we cannot have $B_{2} \subseteq B_{1}$, because that would imply $B_{2}\left(x_{2}\right) \leq$ $B_{1}\left(x_{2}\right)$.

So, we indeed have $B_{1} \nsubseteq \subseteq B_{2}$ and $B_{2} \nsubseteq B_{1}$.

$2.2^{\circ}$. Let us now consider the case when $A$ is a 2-element crisp set, i.e., when $A=\left\{x_{0}, x_{1}\right\}$.

In this case, we can take $B_{1}=\left\{x_{0}\right\}$ and $B_{2}=\left\{x_{1}\right\}$. Clearly, $B_{1} \subseteq A$ and $B_{2} \subseteq A$, but $B_{1} \nsubseteq B_{2}$ and $B_{2} \nsubseteq B_{1}$.

So, the proposition is proven.

Proposition 3. A normalized fuzzy set $A$ is a crisp 2-element set if and only if the following two conditions are satisfied:

- the set $A$ itself is not a 1-element crisp set and not a 2-element non-crisp set, but

- each proper normalized fuzzy subsets $B \subseteq A$ is either a crisp 1-element sets or a non-crisp 2-element set.

\section{Proof.}

$1^{\circ}$. If $A$ is a 2-element crisp set, i.e., if $A=\left\{x_{0}, x_{1}\right\}$ for some $x_{0} \neq x_{1}$, then it is clearly:

- not a 1-element crisp set, and

- not a non-crisp 2-element set.

Let us prove that in this case, every proper normalized fuzzy subset $B \subseteq A$ is

- either a 1-element crisp set

- or a non-crisp 2-element set.

Since $A(x)>0$ for only two values $x=x_{0}$ and $x=x_{1}$, and $B(x) \leq A(x)$ for all $x$, the value $B(x)$ can be positive also for at most two values $x_{i}$.

If $B(x)>0$ for only one value $x$, then, since $B$ is normalized, for this $x$, we must have $B(x)=1$. Thus, we have $B=\{x\}$, i.e., $B$ is a 1 -element crisp set. 
If $B(x)>0$ for two different values $x$, this means that we have $B\left(x_{0}\right)>0$ and $B\left(x_{1}\right)>0$. Since the set $B$ is normalized, one of these value must be equal to 1 . If the second one is equal to 1 , we will have $B=A-$ but $B$ is a proper subset. Thus, one of the values $B\left(x_{i}\right)$ is smaller than 1 - thus, $B$ is a non-crisp 2-element set.

$2^{\circ}$. Let us now prove that if a normalized fuzzy set $A$ is not a 2-element crisp set, then one of the above properties is not satisfied, i.e.,

- either $A$ is 1 -element crisp set or a 2-element non-crisp set,

- or one of its proper subsets $B \subseteq A$ is not a non-crisp 2-element set.

In other words, we want to prove that if $A$ is:

- not a crisp 1-element set,

- not a crisp 2-element set, and

- not a non-crisp 2-element set,

then one of its proper subsets $B \subseteq A$ is not a non-crisp 2element set.

The condition on $A$ means that it is:

- not a 1-element set and

- not a 2-element set.

This means that there must exist at least three different values $x \in U$ for which $A(x)>0$. For one of these values, we have $A\left(x_{0}\right)=1$, let us denote the other two values by $x_{1}$ and $x_{2}$, then $A\left(x_{1}\right)>0$ and $A\left(x_{2}\right)>0$.

Let us now take the following normalized fuzzy set $B$;

- $B\left(x_{1}\right)=0.5 \cdot A\left(x_{1}\right)$,

- $B\left(x_{2}\right)=0.5 \cdot A\left(x_{2}\right)$, and

- $B(x)=A(x)$ for all other $x$.

Here, $B\left(x_{0}\right)=A\left(x_{0}\right)=1$, so $B$ is indeed a normalized fuzzy set. One can easily check that $B(x) \leq A(x)$ for all $x$, so it is indeed a subset of $A$. Since $A\left(x_{1}\right)>0$, we have $B\left(x_{1}\right)=0.5 \cdot A\left(x_{1}\right) \neq A\left(x_{1}\right)$, so $B$ is a proper subset of $A$.

However, $B\left(x_{0}\right)=1>0, B\left(x_{1}\right)>0$, and $B\left(x_{2}\right)>0$, so $B$ is not a 2-element set.

The proposition is proven.

Comment. Now, we are ready to show that crisp sets can be described in terms of the subsethood relation.

Proposition 4. A normalized fuzzy set is crisp if and only if we have one of the following two cases:

- A is a 1-element fuzzy set, or

- for every subset $B \subseteq A$ which is a non-crisp 2-element set, there exists a crisp 2-element set $C$ for which

$$
B \subseteq C \subseteq A
$$

Comment. Since Propositions 1-3 show that the properties of being a crisp 1-element set, a crisp 2-element set, and a non-crisp 2-element set can all be described in terms of the subsethood relation, this Proposition shows that crispness can indeed be described in terms of subsethood.

\section{Proof.}

$1^{\circ}$. Let us first prove that if $A$ is a crisp set, then:

- either it is a 1-element crisp set,

- or for every non-crisp 2-element set $B \subseteq A$, there exists a crisp 2-element set $C$ for which $B \subseteq C \subseteq A$.

Indeed, let $B$ be a non-crisp 2-element set. This means that for some elements $x_{0} \in U$ and $x_{1} \in U$, we have:

- $B\left(x_{0}\right)=1$,

- $0<B\left(x_{1}\right)<1$, and

- $B(x)=0$ for all other $x$.

Since $B \subseteq A$, we have:

- $1=B\left(x_{0}\right) \leq A\left(x_{0}\right)-$ thus $A\left(x_{0}\right)=1$; and

- $0<B\left(x_{2}\right) \leq A\left(x_{1}\right)$ - thus $A\left(x_{1}\right)>0$.

The set $A$ is crisp, so $A\left(x_{1}\right)$ can be either 0 or 1 . Since $A\left(x_{1}\right)>0$, we must have $A\left(x_{1}\right)=1$. Thus, for a 2-element crisp set $C=\left\{x_{0}, x_{1}\right\}$, we have $B \subseteq C \subseteq A$.

$2^{\circ}$. To complete our proof, let us prove that if a normalized crisp set $A$ is not a crisp set, then there exists a non-crisp 2-element set $B \subseteq A$ for which no crisp 2-element set $C$ satisfies the property $B \subseteq C \subseteq A$.

By definition, for a crisp set, all the values $A(x)$ are either 0 s or $1 \mathrm{~s}$. So, the fact that $A$ is not crisp means that we have $0<A\left(x_{1}\right)<1$ for some $x_{1} \in U$.

Since $A$ is normalized, there exists $x_{0}$ for which $A\left(x_{0}\right)=1$. Let us now take the following set $B$;

- $B\left(x_{0}\right)=1$,

- $0<B\left(x_{1}\right)=A\left(x_{1}\right)<1$, and

- $B(x)=0$ for all other $x$.

Clearly, $B$ is a non-crisp 2-element set a nd $B \subseteq A$.

If we had $B \subseteq C \subseteq A$ for some crisp 2-element set $C$, then due to $1=B\left(x_{0}\right) \leq C\left(x_{0}\right)$ and $B\left(x_{1}\right) \leq C\left(x_{1}\right)$, we would have $C\left(x_{0}\right)=1$ and $C\left(x_{1}\right)>0-$ hence $C\left(x_{1}\right)=1$ (since $C$ is crisp). But in this case, $C\left(x_{1}\right)=1>A\left(x_{1}\right)$, so we cannot have $C \subseteq A$.

The proposition is proven.

\section{INTERVAL-VALUED CASE}

Formulation of the problem. The traditional fuzzy logic assumes that experts can meaningfully describe their degrees of certainty by numbers from the interval $[0,1]$. In practice, however, experts cannot meaningfully select a single number fuzzy describing their degree of certainty - since it is not possible to distinguish between, say, degrees 0.80 and 0.81 . A more adequate description of the expert's uncertainty is when we allow to characterize the uncertainty by a whole range of possible numbers, i.e., by an interval $[\underline{A}(x), \bar{A}(x)]$.

This idea leads to interval-valued fuzzy numbers (see, e.g., [3], [4]), i.e., mappings that assign, to each element $x$ from the Universe of discourse, an interval $A(x)=[\underline{A}(x), \bar{A}(x)]$.

For two interval-valued degrees $A=[\underline{A}, \underline{\bar{A}}]$ and $B=$ $[\underline{B}, \bar{B}]$, it is reasonable to say that $A \leq B$ if

$$
\underline{A} \leq \underline{B} \text { and } \bar{A} \leq \bar{B} \text {. }
$$


Thus, we can define a subsethood relation between two interval-valued fuzzy sets $A$ and $B$ as $A(x) \leq B(x)$ for all $x$.

An interval-valued fuzzy set is normalized if $\bar{A}\left(x_{0}\right)=1$ for some $x_{0}$.

Traditional (type-2) fuzzy sets can be viewed as particular cases of interval-valued fuzzy sets, with "degenerate" intervals

$$
[A(x), A(x)] \text {. }
$$

Here, we have a similar problem: can we detect traditional fuzzy sets based only on the subsethood relation between interval-valued fuzzy sets?

Let us show that this is indeed possible.

Definition 2. By an uncertain 1-element set, we mean a normalized interval-valued fuzzy set $A$ for which, for some $x_{0} \in U$, we have:

- $A\left(x_{0}\right)=[0,1]$ and

- $A(x)=[0,0]$ for all other $x$.

Proposition 5. A normalized interval-valued fuzzy set $A$ is an uncertain 1-element set if and only if it has no proper normalized subsets.

Comment. So, we can determine uncertain 1-element sets based on the subsethood relation.

\section{Proof.}

$1^{\circ}$. Let us first prove that for an uncertain 1-element set $A$, there are no proper subsets.

Indeed, if $A\left(x_{0}\right)=[0,1], A(x)=[0,0]$ for all $x \neq x_{0}$, and $B(x) \leq A(x)$, then:

- for $x \neq x_{0}$, from $\underline{B}(x) \leq \underline{A}(x)=0$ and $\bar{B}(x) \leq \bar{A}(x)=$ 0 , it follows that $\underline{B}(x)=\overline{\bar{B}}(x)=0$, so

$$
B(x)=[0,0]=A(x) ;
$$

- for $x=x_{0}$, from $\underline{A}\left(x_{0}\right) \leq \bar{A}\left(x_{0}\right)=0$, it follows that

$$
\underline{B}\left(x_{0}\right)=0=\underline{A}\left(x_{0}\right) \text {. }
$$

On the other hand, $B$ is a normalized interval-valued fuzzy set, so we must have loverline $B(x)=1$ for some $x$. This cannot be for $x \neq x_{0}$, since then $\bar{B}(x)=0$. So, the only remaining option is $x=x_{0}$. Hence, $\bar{B}\left(x_{0}\right)=1$, thus, $\bar{B}\left(x_{0}\right)=\bar{A}\left(x_{0}\right)$.

Therefore, if $B \subseteq A$, then $B=A$. So, the normalized interval-valued fuzzy sets $A$ does not have any proper subsets.

$2^{\circ}$. To complete the proof, let us prove that if a normalized interval-valued fuzzy set has no proper subsets, then it is an uncertain 1-element set.

Indeed, since $A$ is normalized, there exists an element $x_{0}$ for which $\bar{A}\left(x_{0}\right)=1$. Then, as one can easily check, we have $B \subseteq A$, where:

- $B\left(x_{0}\right)=[0,1]$, and

- $B(x)=[0,0]$ for all other $x$

Since $A$ has no proper subsets, we thus conclude that $A=B$, i.e., that $A$ is an uncertain 1-element set.
The proposition is proven.

Definition 3. By a basic 1-element set, we mean a normalized interval-valued fuzzy set $A$ for which, for some $x_{0} \in U$, we have:

- $A\left(x_{0}\right)=[a, 1]$ for some $a>0$, and

- $A(x)=[0,0]$ for all $x \neq x_{0}$.

Definition 4. By a basic 2-element set, we mean a normalized interval-valued fuzzy set $A$ for which, for some $x_{0} \neq x_{1}$, we have:

- $A\left(x_{0}\right)=[0,1]$,

- $A\left(x_{1}\right)=[0, a]$ for some $a \in(0,1)$, and

- $A(x)=[0,0]$ for all other $x$.

Proposition 6. Let $A$ be a normalized interval-valued fuzzy set which is not an uncertain 1-element set. Then, the following two conditions are equivalent to each other:

- the class $\{B: B \subseteq A\}$ of all subsets of $A$ is linearly ordered;

- A is either a basic 1-element set or a basic 2-element set.

Comment. So, we can determine, based on the subsethood relation, whether $A$ is a basic set.

\section{Proof.}

$1^{\circ}$. Let us first prove that if $A$ is a basic 1-element set or a basic 2-element set, then the class of all its subsets is linearly ordered.

$1.1^{\circ}$. Let us first consider the case when $A$ is a basic 1 -element set.

In this case, $B \subseteq A$ implies $\underline{B}(x)=\bar{B}(x)=0$ for all $x \neq x_{0}$. Since $B$ is normalized, then, similarly to the proof of Proposition 5 , we get $\bar{B}\left(x_{0}\right)=1$. The final inequality $\underline{B}\left(x_{0}\right) \leq \underline{A}\left(x_{0}\right)=a$ implies that for $b \stackrel{\text { def }}{=} \underline{B}\left(x_{0}\right)$, we have

$$
b \leq a
$$

So, the set $B$ has the following form:

- $B(x)=[0,0]$ for all $x \neq x_{0}$, and

- $B\left(x_{0}\right)=[b, 1]$, where we denoted $b=\underline{B}\left(x_{0}\right)$.

One can easily check that the class of such sets is linearly ordered: namely, if for two such sets $B_{1}$ and $B_{2}$, we denote the corresponding values $b$ by $b_{1}$ and $b_{2}$, then:

- if $b_{1} \leq b_{2}$, then $B_{1} \subseteq B_{2}$, and

- vice versa, if $b_{2} \leq b_{1}$, then $B_{2} \subseteq B_{1}$.

$1.2^{\circ}$. Let us consider the case when $A$ is a basic 2-element set.

Let $B \subseteq A$. Then, from $B(x) \leq A(x)$, we conclude that $B(x)=[0,0]$ when $x$ is different from $x_{0}$ and $x_{1}$, and that $\underline{B}\left(x_{0}\right)=\underline{B}\left(x_{1}\right)=0$.

The set $B$ is normalized, so $\bar{B}(x)=1$ for some $x$.

- This $x$ cannot be different from $x_{0}$ and $x_{1}$, since for such $x$, we have

$$
\bar{B}(x)=0<1 \text {. }
$$


- It cannot be equal to $x_{1}$, since we have

$$
\bar{B}\left(x_{1}\right) \leq \bar{A}\left(x_{1}\right)=a<1 \text {. }
$$

Thus, the only possible element $x$ is $x=x_{0}$, hence we have $\bar{B}\left(x_{0}\right)=1$. The final inequality $\bar{B}\left(x_{1}\right) \leq \bar{A}\left(x_{1}\right)=a$ implies that for $b \stackrel{\text { def }}{=} \bar{B}\left(x_{1}\right)$, we have $b \leq a$.

So, the set $B$ has the following form:

- $B(x)=[0,0]$ for all $x$ which are different from $x_{0}$ and $x_{1}$;

- $B\left(x_{0}\right)=[0,1]$, and

- $B\left(x_{1}\right)=[0, b]$, where $b=\bar{B}\left(x_{1}\right)$.

One can easily check that the class of such sets is linearly ordered: namely, if for two such sets $B_{1}$ and $B_{2}$, we denote the corresponding values $b$ by $b_{1}$ and $b_{2}$, then:

- if $b_{1} \leq b_{2}$, then $B_{1} \subseteq B_{2}$, and

- vice versa, if $b_{2} \leq b_{1}$, then $B_{2} \subseteq B_{1}$.

$2^{\circ}$. Let us now prove that if the class of all normalized subsets of a normalized fuzzy interval-valued set $A$ is linearly ordered, then $A$ is either a basic 1-element set or a basic 2-element set.

Since the set $A$ is normalized, there exists an element $x_{0} \in U$ for which $\bar{A}\left(x_{0}\right)=1$. Let us consider two possible cases:

- $\underline{A}\left(x_{0}\right)>0$ and

- $\underline{A}\left(x_{0}\right)=0$.

$2.1^{\circ}$. Let us first consider he case when $\underline{A}\left(x_{0}\right)>0$. Let us prove that in this case, we have a basic 1-element set, i.e., that $A(x)=[0,0]$ for all $x \neq x_{0}$.

We will prove this by contradiction. Let us assume that $\bar{A}(x)>0$ for some $x \neq x_{0}$. Then, we can consider the following two subsets of $A$ :

- $B_{1}\left(x_{0}\right)=A\left(x_{0}\right), B_{2}\left(x_{0}\right)=[0,1]$;

- $B_{2}\left(x_{1}\right)=[0,0], B_{2}\left(x_{1}\right)=A\left(x_{1}\right)$, and

- $A(x)=B(x)=[0,0]$ for al other $x \in U$.

One can easily check that $B_{1} \subseteq A$ and $B_{2} \subseteq A$. However:

- we have $\underline{B}_{1}\left(x_{0}\right)=\underline{A}\left(x_{0}\right)>0=\underline{B}_{2}\left(x_{0}\right)$, hence we cannot have $B_{1} \subseteq B_{2}$;

- on the other hand, $\bar{B}_{2}\left(x_{1}\right)=\bar{A}\left(x_{1}\right)>0=\bar{B}_{1}\left(x_{1}\right)$, hence we cannot have $B_{2} \subseteq B_{1}$.

The fact that here $B_{1} \nsubseteq B_{2}$ and $B_{2} \nsubseteq B_{1}$ shows that $\bar{A}(x)>0$ is impossible. Thus, $\bar{A}(x)=0$ for all $x \neq x_{0}$, so $A$ is indeed a basic 1-element set.

$2.2^{\circ}$. Let us first consider he case when $\underline{A}\left(x_{0}\right)=0$. Let us prove that in this case, we have a basic 2-element set, i.e., that:

- $A\left(x_{1}\right)=[0, a]$ for some $x_{1} \in U$ and some $a \in(0,1)$, and

- $A(x)=[0,0]$ for all other $x$.

Indeed, since $A\left(x_{0}\right)=[0,1]$, but the set $A$ is not an uncertain 1-element set, there exists some $x_{1} \neq x_{0}$ for which $\bar{A}\left(x_{1}\right)>0$.
$2 \cdot 2 \cdot 1^{\circ}$. Let us prove that in this case, $A(x)=[0,0]$ for all other $x$.

We prove this by contradiction. Let us assume that for some $x_{2}$, we have $x_{2} \neq x_{0}, x_{2} \neq x_{1}$ and $\bar{A}\left(x_{2}\right)>0$. In this case, we can form the following two subsets $B_{1}$ and $B_{2}$;

- $B_{1}\left(x_{0}\right)=B_{2}\left(x_{0}\right)=[0,1]$;

- $B_{1}\left(x_{1}\right)=A\left(x_{1}\right), B_{2}\left(x_{1}\right)=[0,0]$;

- $B_{1}\left(x_{2}\right)=[0,0], B_{2}\left(x_{2}\right)=A\left(x_{2}\right)$; and

- $B_{1}(x)=B_{2}(x)=[0,0]$ or all other $x$.

Clearly, $B_{1} \subseteq A$ and $B_{2} \subseteq A$, but:

- $\bar{B}_{1}\left(x_{1}\right)>0=\bar{B}_{2}\left(x_{1}\right)$, so we cannot have $B_{1} \subseteq B_{2}$, and

- $\bar{B}_{2}\left(x_{2}\right)=\bar{A}\left(x_{2}\right)>0=\underline{B}_{1}\left(x_{2}\right)$, so we cannot have

$$
B_{2} \subseteq B_{1} \text {. }
$$

This contradicts to our assumption that the class of all subsets of $A$ is linearly ordered. Thus, $A(x)=[0,0]$ for all element $x$ which are different from $x_{0}$ and $x_{1}$.

$2.2 .2^{\circ}$. Let us prove, by contradiction, that $\underline{A}\left(x_{1}\right)=0$.

Indeed, if $\underline{A}\left(x_{1}\right)>0$, then we can form the following sets $B_{1}$ and $B_{2}$ :

- $B_{1}\left(x_{0}\right)=B_{2}\left(x_{0}\right)=[0,1]$;

- $B_{1}\left(x_{1}\right)=\left[0, \bar{A}\left(x_{1}\right)\right], B_{2}\left(x_{1}\right)=0.5 \cdot \underline{A}\left(x_{1}\right)$.

- $B_{1}(x)=B_{2}(x)=[0,0]$ for all other $x$.

One can easily check that $B_{1} \subseteq A$ and $B_{2} \subseteq A$, but:

- $\bar{B}_{1}\left(x_{1}\right)=\bar{A}\left(x_{1}\right) \geq \underline{A}\left(x_{1}\right)>0.5 \cdot \underline{A}\left(x_{1}\right)=\bar{B}_{2}\left(x_{1}\right)$, so we do not have $B_{1} \subseteq B_{2}$;

- on the other hand, $\underline{B}_{2}\left(x_{1}\right)=0.5 \cdot \underline{A}\left(x_{1}\right)>0=\underline{B}_{1}\left(x_{1}\right)$, so we do not have $B_{2} \subseteq B_{1}$ either.

This contradicts to our assumption that the class of all subsets of $A$ is linearly ordered. This contradiction shows that

$$
\underline{A}_{1}\left(x_{1}\right)=0 \text {. }
$$

\section{$2.2 .3^{\circ}$. Finally, let us prove that $\bar{A}\left(x_{1}\right)<1$.}

Indeed, if $\bar{A}\left(x_{1}\right)=1$, i.e., if $A\left(x_{1}\right)=[0,1]$, then we can find the following two sets $B_{1} \subseteq A$ and $B_{2} \subseteq A$ for which $B_{1} \nsubseteq \nsubseteq B_{2}$ and $B_{2} \nsubseteq B_{1}$ :

- $B_{1}\left(x_{0}\right)=[0,1], B_{2}\left(x_{0}\right)=[0,0]$;

- $B_{1}\left(x_{1}\right)=[0,0], B_{2}\left(x_{1}\right)=A\left(x_{1}\right)=[0,1]$, and

- $B_{1}(x)=B_{2}(x)=[0,0]$ for all other $x$.

Then:

- $\bar{B}_{1}\left(x_{0}\right)=1>\bar{B}_{2}\left(x_{0}\right)$, so we cannot have $B_{1} \subseteq B_{2}$;

- $\bar{B}_{2}\left(x_{1}\right)=1>0=\bar{B}_{1}\left(x_{1}\right)$, so we cannot have $\bar{B}_{2} \subseteq B_{1}$. Contradiction show that we cannot have $\bar{A}\left(x_{1}\right)=1$, thus

$$
\bar{A}\left(x_{1}\right)<1 \text {. }
$$

Thus, in this case, $A$ is a basic 2-element set. The proposition is proven. 
Proposition 7. If $A$ is a basic 1-element set or a basic 2element set, then the following two properties are equivalent to each other:

- A is a crisp 1-element set;

- no proper superset of $A$ is a basic 1-element set or a basic 2-element set.

Comment. So, we can determine crisp 1-element sets based only on the subsethood relation.

Proof. If $A=\left\{x_{0}\right\}$, then clearly $A$ cannot have any proper supersets which are basic 1-element or basic 2-element sets.

Vice versa, if $A$ is a basic 1-element set with $\underline{A}\left(x_{0}\right)<1$, then $B=\left\{x_{0}\right\}$ is its proper superset which is a a 1-element basic set.

Similarly, if $A$ is a basic 2-element set, with $A\left(x_{0}\right)=[0,1]$. $\underline{A}\left(x_{1}\right)=0$, and $\bar{A}\left(x_{1}\right)<1$, then we can have the following proper superset $B \supseteq A$ wit is also a basic 2-element set:

- $B\left(x_{0}\right)=[0,1]$;

- $B\left(x_{1}\right)=\left[0, \frac{1+\bar{A}\left(x_{1}\right)}{2}\right]$; and

- $B(x)=0$ for all other $x$.

The proposition is proven.

Proposition 8. For a normalized interval-valued fuzzy set, the following two conditions are satisfied:

- $A$ is either an uncertain 1-element set or a basic 1element set;

- $A$ is a subset of a crisp 1-element set.

Proof: straightforward.

Comment. Since we know how to describe, based on the subsethood relation,

- when $A$ is an uncertain 1-element set, and

- when $A$ is a basic set,

we can therefore determine:

- basic 1-element sets and

- basic 2-element sets

based on subsethood relation only.

Definition 5. Let $A$ be a basic 2-element set, with:

- $A\left(x_{0}\right)=[0,1]$,

- $A\left(x_{1}\right)=[0, a]$ for some $a \in(0,1)$, and

- $A(x)=[0,0]$ for all other $x$.

Then, by its type-1 cover, we mean a normalized intervalvalued fuzzy set $A^{\prime}$ for which:

- $A^{\prime}\left(x_{0}\right)=[1,1]$,

- $A^{\prime}\left(x_{1}\right)=[a, a]$, and

- $A^{\prime}(x)=[0,0]$ for all other $x$.

Let us show that the type- 1 cover can be determined in terms of the subsethood relation.

Proposition 9. Let $A$ be a basic 2-element set. Then, its type-1 cover $A^{\prime}$ is the $\subseteq$-smallest normalized interval-valued fuzzy set that contains all the normalized interval-valued sets $B \supseteq A$ for which the following four conditions are satisfied:
- the set B is not a basic 2-element set;

- the class of all basic 2-element subsets of B is linearly ordered;

- the class $\{C: A \subseteq C \subseteq B\}$ of all normalized intervalvalued set between $A$ and $B$ is linearly ordered; and

- the set B has only one uncertain 1-element subset.

Proof.

$1^{\circ}$. Let us first prove that a set $B$ satisfies the above four conditions if and only if it has one the following two forms:

- either it has the form $B\left(x_{0}\right)=[b, 1]$ for some $b>0$, $B\left(x_{1}\right)=A\left(x_{1}\right)$, and $B(x)=[0,0]$ for all other $x$; we will call these $B$ of the first form;

- or it has the form $B\left(x_{0}\right)=A\left(x_{0}\right), B\left(x_{1}\right)=[b, a]$ for some $b>0$, and $B(x)=[0,0]$ for all other $x$; we will call these $B$ of the second form.

$1.1^{\circ}$. Let us first prove that the all the sets $B$ of the first form satisfy all the above four conditions.

1.1.1 ${ }^{\circ}$. Indeed, clearly, such $B$ is not a basic 2-element set.

1.1.2 . If $C$ is a basic 2-element set for which $C \subseteq B$, then we have:

- $C\left(x_{0}\right)=[0,1]$,

- $C(x)=[0,0]$ for all $x$ different from $x_{0}$ and $x_{1}$, and

- $C\left(x_{1}\right)=[0, c]$ for some $c \leq a$.

Clearly, the set of all such $C$ is linearly ordered: if we have two such sets, corresponding to elements $c_{1}$ and $c_{2}$, then:

- if $c_{1} \leq c_{2}$, then we have $C_{1} \subseteq C_{2}$, and

- if $c_{2} \leq c_{1}$, then we have $C_{2} \subseteq C_{1}$.

$1.1 .3^{\circ}$. If $A \subseteq C \subseteq B$, then we have:

- $C\left(x_{0}\right)=[c, 1]$ for some $c \in[b, 1]$,

- $C\left(x_{1}\right)=A\left(x_{1}\right)$, and

- $C(x)=[0,0]$ for all other $x$.

Thus, if we have two such sets, corresponding to elements $c_{1}$ and $c_{2}$, then:

- if $c_{1} \leq c_{2}$, then we have $C_{1} \subseteq C_{2}$, and

- if $c_{2} \leq c_{1}$, then we have $C_{2} \subseteq C_{1}$.

1.1.4 $4^{\circ}$. Of course, the only uncertain 1-element set contained in $B$ is the set corresponding to $x_{0}$.

All four conditions are proven.

$1.2^{\circ}$. Let us now prove that the all the sets $B$ of the second form satisfy all the above four conditions.

1.2.1 $1^{\circ}$. Indeed, clearly, such $B$ is not a basic 2-element set.

1.2.2 . If $C \subseteq B$ is a basic 2-element set, then we have:

- $C\left(x_{0}\right)=[0,1]$,

- $C(x)=[0,0]$ for all $x$ different from $x_{0}$ and $x_{1}$, and

- $C\left(x_{1}\right)=[0, c]$ for some $c \leq a$.

Clearly, the set of all such $C$ is linearly ordered: if we have two such sets, corresponding to elements $c_{1}$ and $c_{2}$, then:

- if $c_{1} \leq c_{2}$, then we have $C_{1} \subseteq C_{2}$, and 
- if $c_{2} \leq c_{1}$, then we have $C_{2} \subseteq C_{1}$.

1.2.3 . If $A \subseteq C \subseteq B$, then we have:

- $C\left(x_{0}\right)=A\left(x_{0}\right)$,

- $C\left(x_{1}\right)=[c, a]$ for some $c \in[b, a]$, and

- $C(x)=[0,0]$ for all other $x$.

Thus, if we have two such sets, corresponding to elements $c_{1}$ and $c_{2}$, then:

- if $c_{1} \leq c_{2}$, then we have $C_{1} \subseteq C_{2}$, and

- if $c_{2} \leq c_{1}$, then we have $C_{2} \subseteq C_{1}$.

$1.2 .4^{\circ}$. Of course, the only uncertain 1 -element set contained in $B$ is the set corresponding to $x_{0}$.

All four conditions are proven.

$1.3^{\circ}$. Let us now prove that if a set $B$ satisfies the above four conditions, then $B$ is either of the first form or of the second form.

$1.3 .1^{\circ}$. Let us first prove that we must have $B(x)=[0,0]$ for all elements $x$ which are different from $x_{0}$ and $x_{1}$.

We will prove this by contradiction. Assume that $\bar{B}\left(x_{2}\right)>0$ for some element $x_{2}$ which is different from $x_{0}$ and $x_{1}$. Then, in addition to a basic 2-element set $A \subseteq B$, we also have another basic 2-element set $C \subseteq B$ for which:

- $C\left(x_{0}\right)=[0,1]$,

- $C\left(x_{2}\right)=\left[0, \bar{B}\left(x_{2}\right)\right]$, and

- $C(c)=[0,0]$ for all other elements $x$.

Then:

- $\bar{A}\left(x_{1}\right)=a>0=\bar{C}\left(x_{1}\right)$, so we cannot have $A \subseteq C$; and

- $\bar{C}\left(x_{2}\right)>0=\bar{A}\left(x_{2}\right)$, so we cannot have $C \subseteq A$ either.

This contradicts to the condition that set of all basic 2-element sets which are subsets of $B$ is linearly ordered.

Thus, $\bar{B}(x)>0$ is impossible. So, indeed, $B(x)=[0,0]$ for all elements $x$ which are different from $x_{0}$ and $x_{1}$.

1.3.2 $2^{\circ}$. Due to Part 1.3.1 of this proof, the set $B$ is uniquely described by its values $B\left(x_{0}\right)$ and $B\left(x_{1}\right)$. The condition that $A \subseteq B$ implies that $\bar{A}\left(x_{0}\right)=1$ and that:

- $\bar{B}\left(x_{0}\right) \geq 0$,

- $\underline{B}\left(x_{1}\right) \geq 0$, and

- that $\bar{B}\left(x_{1}\right) \geq a=\bar{A}\left(x_{1}\right)$.

Since $B$ is not a basic 2-element set and $A$ is such a set, we have $B \neq A$. Thus, at least one of the above inequalities must be strict. Let us consider these three inequalities one by one.

$1.3 .3^{\circ}$. Let us first consider the case when $\bar{B}\left(x_{0}\right)>0$. Let us prove that in this case, we have $B\left(x_{1}\right)=A\left(x_{1}\right)$, i.e., that we have a set of the first form.

We will first prove, by contradiction, that $\underline{B}\left(x_{1}\right)=0$. Indeed, if $\underline{B}\left(x_{1}\right)>0$, then we can form the following two sets $C_{1}$ and $C_{2}$ for which $A \subseteq C_{1} \subseteq B, A \subseteq C_{2} \subseteq B$, but $C_{1} \nsubseteq C_{2}$ and $C_{2} \nsubseteq C_{1}$ :

- $C_{1}\left(x_{0}\right)=A\left(x_{0}\right)=[0,1], C_{1}\left(x_{1}\right)=B\left(x_{1}\right)$, and $C_{1}(x)=[0,0]$ for all other $x$;
- $C_{2}\left(x_{0}\right)=B\left(x_{0}\right), C_{2}\left(x_{1}\right)=A\left(x_{1}\right)$, and $C_{2}(x)=[0,0]$ for all other $x$.

Here:

- $\underline{C}_{1}\left(x_{1}\right)=\underline{B}\left(x_{1}\right)>0=\underline{C}_{2}\left(x_{1}\right)$, so we cannot have

$$
C_{1} \subseteq C_{2}
$$

- $\underline{C}_{2}\left(x_{0}\right)=\underline{B}\left(x_{0}\right)>0=\underline{C}_{1}\left(x_{0}\right)$, so we cannot have

$$
C_{2} \subseteq C_{1} \text {. }
$$

This contradicts to our assumption that the class of all intermediate fuzzy sets $C$ is linearly ordered. Thus, we must have $\underline{B}\left(x_{1}\right)=0$.

Let us now prove, by contradiction, that $\bar{B}\left(x_{1}\right)=\bar{A}\left(x_{1}\right)$. Indeed, suppose that $\bar{B}\left(x_{1}\right)>\bar{A}\left(x_{1}\right)$. Then we can form the following two sets $C_{1}$ and $C_{2}$ for which $A \subseteq C_{1} \subseteq B, A \subseteq$ $C_{2} \subseteq B$, but $C_{1} \nsubseteq C_{2}$ and $C_{2} \nsubseteq C_{1}$ :

- $C_{1}\left(x_{0}\right)=A\left(x_{0}\right)=[0,1], C_{1}\left(x_{1}\right)=B\left(x_{1}\right)$, and $C_{1}(x)=[0,0]$ for all other $x$;

- $C_{2}\left(x_{0}\right)=B\left(x_{0}\right), C_{2}\left(x_{1}\right)=A\left(x_{1}\right)$, and $C_{2}(x)=[0,0]$ for all other $x$.

Here:

- $\bar{C}_{1}\left(x_{1}\right)=\bar{B}\left(x_{1}\right)>\bar{A}\left(x_{1}\right)=\bar{C}_{2}\left(x_{1}\right)$, so we cannot have $C_{1} \subseteq C_{2}$

- $\underline{C}_{2}\left(x_{0}\right)=\underline{B}\left(x_{0}\right)>0=\underline{C}_{1}\left(x_{0}\right)$, so we cannot have

$$
C_{2} \subseteq C_{1} \text {. }
$$

This contradicts to our assumption that the class of all intermediate fuzzy sets $C$ is linearly ordered. Thus, we must have

$$
\bar{B}\left(x_{1}\right)=\bar{A}\left(x_{1}\right) \text {. }
$$

So, in this case, we indeed have a set of the first form.

$1.3 .4^{\circ}$. Let us now consider the case when $\underline{B}\left(x_{1}\right)>0$. Let us prove that in this case, we have $\bar{B}\left(x_{0}\right)=0$ and $\bar{B}\left(x_{1}\right)=$ $\bar{A}\left(x_{1}\right)$, i.e., that we have a set of the second form.

We will first prove, by contradiction, that $\underline{B}\left(x_{0}\right)=0$. Indeed, if $\underline{B}\left(x_{0}\right)>0$, then we can form the following two sets $C_{1}$ and $C_{2}$ for which $A \subseteq C_{1} \subseteq B, A \subseteq C_{2} \subseteq B$, but $C_{1} \nsubseteq C_{2}$ and $C_{2} \nsubseteq C_{1}$ :

- $C_{1}\left(x_{0}\right)=A\left(x_{0}\right)=[0,1], C_{1}\left(x_{1}\right)=B\left(x_{1}\right)$, and $C_{1}(x)=[0,0]$ for all other $x$;

- $C_{2}\left(x_{0}\right)=B\left(x_{0}\right), C_{2}\left(x_{1}\right)=A\left(x_{1}\right)$, and $C_{2}(x)=[0,0]$ for all other $x$.

Here:

- $\underline{C}_{1}\left(x_{1}\right)=\underline{B}\left(x_{1}\right)>0=\underline{C}_{2}\left(x_{1}\right)$, so we cannot have

$$
C_{1} \subseteq C_{2}
$$

- $\underline{C}_{2}\left(x_{0}\right)=\underline{B}\left(x_{0}\right)>0=\underline{C}_{1}\left(x_{0}\right)$, so we cannot have

$$
C_{2} \subseteq C_{1} \text {. }
$$


This contradicts to our assumption that the class of all intermediate fuzzy sets $C$ is linearly ordered. Thus, we must have

$$
\underline{B}\left(x_{0}\right)=0 \text {. }
$$

Let us now prove, by contradiction, that $\bar{B}\left(x_{1}\right)=\bar{A}\left(x_{1}\right)$. Indeed, suppose that $\bar{B}\left(x_{1}\right)>\bar{A}\left(x_{1}\right)$. Then we can form the following two sets $C_{1}$ and $C_{2}$ for which $A \subseteq C_{1} \subseteq B, A \subseteq$ $C_{2} \subseteq B$, but $C_{1} \nsubseteq C_{2}$ and $C_{2} \nsubseteq C_{1}$ :

- $C_{1}\left(x_{0}\right)=[0,1], C_{1}\left(x_{1}\right)=B\left(x_{1}\right)$, and $C_{1}(x)=[0,0]$ for all other $x$;

- $C_{2}\left(x_{0}\right)=B\left(x_{0}\right), C_{2}\left(x_{1}\right)=A\left(x_{1}\right)$, and $C_{2}(x)=[0,0]$ for all other $x$.

Here:

- $\bar{C}_{1}\left(x_{1}\right)=\bar{B}\left(x_{1}\right)>\bar{A}\left(x_{1}\right)=\bar{C}_{2}\left(x_{1}\right)$, so we cannot have

$$
C_{1} \subseteq C_{2}
$$

- $\underline{C}_{2}\left(x_{0}\right)=\underline{B}\left(x_{0}\right)>0=\underline{C}_{1}\left(x_{0}\right)$, so we cannot have

$$
C_{2} \subseteq C_{1}
$$

This contradicts to our assumption that the class of all intermediate fuzzy sets $C$ is linearly ordered. Thus, we must have

$$
\bar{B}\left(x_{1}\right)=\bar{A}\left(x_{1}\right) \text {. }
$$

So, in this case, we indeed have a set of the second form.

$1.3 .5^{\circ}$. Finally, let us prove that the case when $\bar{B}\left(x_{1}\right)>\bar{A}\left(x_{1}\right)$ is not possible.

We will first prove, by contradiction, that in this case, $\underline{B}\left(x_{0}\right)=$ 0 . Indeed, if $\underline{B}\left(x_{0}\right)>0$, then we can form the following two sets $C_{1}$ and $C_{2}$ for which $A \subseteq C_{1} \subseteq B, A \subseteq C_{2} \subseteq B$, but $C_{1} \nsubseteq C_{2}$ and $C_{2} \nsubseteq C_{1}$ :

- $C_{1}\left(x_{0}\right)=A\left(x_{0}\right)=[0,1], C_{1}\left(x_{1}\right)=B\left(x_{1}\right)$, and $C_{1}(x)=[0,0]$ for all other $x$;

- $C_{2}\left(x_{0}\right)=B\left(x_{0}\right), C_{2}\left(x_{1}\right)=A\left(x_{1}\right)$, and $C_{2}(x)=[0,0]$ for all other $x$.

Here:

- $\bar{C}_{1}\left(x_{1}\right)=\bar{B}\left(x_{1}\right)>\bar{A}\left(x_{1}\right)=\bar{C}_{2}\left(x_{1}\right)$, so we cannot have

$$
C_{1} \subseteq C_{2}
$$

- $\underline{C}_{2}\left(x_{0}\right)=\underline{B}\left(x_{0}\right)>0=\underline{C}_{1}\left(x_{0}\right)$, so we cannot have

$$
C_{2} \subseteq C_{1}
$$

This contradicts to our assumption that the class of all intermediate fuzzy sets $C$ is linearly ordered. Thus, we must have

$$
\underline{B}\left(x_{0}\right)=0 \text {. }
$$

Let us now prove, by contradiction, that $\underline{B}\left(x_{1}\right)=0$. Indeed, suppose that $\underline{B}\left(x_{1}\right)>0$. Then we can form the following two sets $C_{1}$ and $C_{2}$ for which $A \subseteq C_{1} \subseteq B, A \subseteq C_{2} \subseteq B$, but $C_{1} \nsubseteq C_{2}$ and $C_{2} \nsubseteq C_{1}$ :

- $C_{1}\left(x_{0}\right)=A\left(x_{0}\right)=[0,1], C_{1}\left(x_{1}\right)=\left[0, \bar{B}\left(x_{1}\right)\right]$, and $C_{1}(x)=[0,0]$ for all other $x$;

- $C_{2}\left(x_{0}\right)=A\left(x_{0}\right)=[0,1], C_{2}\left(x_{1}\right)=\left[\underline{B}\left(x_{1}\right), \bar{A}\left(x_{1}\right)\right]$, and $C_{2}(x)=[0,0]$ for all other $x$.

Here:

- $\bar{C}_{1}\left(x_{1}\right)=\bar{B}\left(x_{1}\right)>\bar{A}\left(x_{1}\right)=\bar{C}_{2}\left(x_{1}\right)$, so we cannot have

$$
C_{1} \subseteq C_{2}
$$

- $\underline{C}_{2}\left(x_{1}\right)=\underline{B}\left(x_{1}\right)>0=\underline{C}_{1}\left(x_{1}\right)$, so we cannot have

$$
C_{2} \subseteq C_{1} \text {. }
$$

This contradicts to our assumption that the class of all intermediate fuzzy sets $C$ is linearly ordered. Thus, we must have

$$
\underline{B}\left(x_{1}\right)=0 \text {. }
$$

Finally, $\bar{B}\left(x_{1}\right)<1$, since otherwise $B$ would have two uncertain 1-element subsets:

- a subset corresponding to $x_{0}$, and

- a subset corresponding to $x_{1}$,

Then, since we know that $\bar{B}\left(x_{0}\right)=1$ and we have proved that $\underline{B}\left(x_{0}\right)=\underline{B}\left(x_{1}\right)=0$ and $\bar{B}\left(x_{1}\right)<1$, we conclude that the set $B$ is a basic 2 -element set - and we explicitly assumed that it is not.

Thus, the third inequality cannot be strict, so $B$ is indeed either of the first form, or of the second form. Once can check that the smallest set containing all such sets is indeed the set $A^{\prime}$.

The proposition is proven.

Definition 6. Let $A$ be an uncertain 1-element set, with:

- $A\left(x_{0}\right)=[0,1]$, and

- $A(x)=[0,0]$ for all other $x$.

Then, by its type-1 cover, we mean a crisp set $A^{\prime}=\left\{x_{0}\right\}$.

Proposition 10. A normalized interval-valued fuzzy set is a type-1 set if and only if the following two conditions are satisfied:

- if $B \subseteq A$ for some uncertain 1-element set, then $B^{\prime} \subset A$, and

- if $B \subseteq A$ for some basic 2-element set, then $B^{\prime} \subseteq A$.

Comment. Since we have shown that:

- the operation $B^{\prime}$,

- uncertain 1-element sets, and

- basic 2-element sets

can all be described in terms of the subsethood relation, we can thus conclude that we can detect type- 1 sets based on the subsethood relation between normalized interval-valued fuzzy sets. 


\section{Proof.}

$1^{\circ}$. One can see that the type- 1 cover of a set $A(x)=$ $[\underline{A}(x), \bar{A}(x)]$ has the form $A^{\prime}(x)=[\bar{A}(x), \bar{A}(x)]$.

For a type-1 set, $\underline{A}(x)=\bar{A}(x)$, thus $A^{\prime}=A$, and clearly, $A \subseteq B$ implies $A^{\prime} \subseteq B$.

$2^{\circ}$. Vice versa, let us prove that if the above two conditions are satisfied, then $A$ is a type- 1 set, i.e., that $\underline{A}(x)=\bar{A}(x)$ for all $x$.

To prove this, let us consider two possible cases:

- elements $x$ for which $\bar{A}(x)=1$, and

- elements $x$ for which $\bar{A}(x)<1$.

$2.1^{\circ}$ Let us first consider an element $x$ for which $\bar{A}(x)=1$. In this case, $B \subseteq A$ for the uncertain 1-element set $B$ for which $B(x)=[0,1]$ and $B(y)=[0,0]$ for all $y \neq x$. Then, $B^{\prime}=\{x\}$, i.e., $B^{\prime}(x)=[1,1]$. Thus, from $B^{\prime} \subseteq A$ it follows that $1=\underline{B}^{\prime}(x) \leq \underline{A}(x)$, so $\underline{A}(x)=1=\bar{A}(x)$. So, for such elements $x$, we indeed have $\underline{A}(x)=\bar{A}(x)$.

$2.2^{\circ}$. Finally, let us consider an element $x$ for which $\bar{A}(x)<1$. Since $A$ is normalized, there exists an element $x_{0}$ for which $\bar{A}\left(x_{0}\right)=1$. Now, we can form the following basic 2-element set $B$ :

- $B\left(x_{0}\right)=[0,1]$,

- $B(x)=[0, \bar{A}(x)]$, and

- $B(y)=[0,0]$ for all other elements $y$.

Clearly, $B \subseteq A$, hence $B^{\prime} \subseteq A$. Here, $B^{\prime}(x)=$ $[\bar{B}(x), \bar{B}(x)]=[\bar{A}(x), \bar{A}(x)]$. So, $B^{\prime} \subseteq A$ implies $\underline{B}^{\prime}(x)=$ $\bar{A}(x) \leq \underline{A}(x)$, thus $\underline{A}(x)=\bar{A}(x)$.

The proposition is proven.

\section{ACKNOWLEDGMENTS}

This work was supported in part by the US National Science Foundation grant HRD-1242122.

The authors are thankful to all the participants of the 2017 Annual Conference of the North American Fuzzy Information Processing Society NAFIPS'2017 (Cancun, Mexico, October $16-18,2017)$ for valuable suggestions.

\section{REFERENCES}

[1] R. Belohlavek, J. W. Dauben, and G. J. Klir, Fuzzy Logic and Mathematics: A Historical Perspective, Oxford University Press, New York, 2017.

[2] G. Klir and B. Yuan, Fuzzy Sets and Fuzzy Logic, Prentice Hall, Upper Saddle River, New Jersey, 1995.

[3] J. M. Mendel, Uncertain Rule-Based Fuzzy Systems: Introduction and New Directions, Springer, Cham, Switzerland, 2017.

[4] J. M. Mendel and D. Wu, Perceptual Computing: Aiding People in Making Subjective Judgments, IEEE Press and Wiley, New York, 2010.

[5] H. T. Nguyen and E. A. Walker, A First Course in Fuzzy Logic, Chapman and Hall/CRC, Boca Raton, Florida, 2006.

[6] V. Novák, I. Perfilieva, and J. Močkoř, Mathematical Principles of Fuzzy Logic, Kluwer, Boston, Dordrecht, 1999.

[7] C. Servin, G. Muela, and V. Kreinovich, "Can we detect crisp sets based only on the subsethood ordering of fuzzy sets? fuzzy sets and/or crisp sets based on subsethood of interval-valued fuzzy sets?", Proceedings of the 2017 Annual Conference of the North American Fuzzy Information Processing Society NAFIPS'2017, Cancun, Mexico, October 16-18, 2017.

[8] L. A. Zadeh, "Fuzzy sets", Information and Control, 1965, Vol. 8, pp. 338-353. 